\title{
AGE-RELATED CHANGES IN THE THICKNESS OF THE CALCIFIED ZONE AND THE NUMBER OF TIDEMARKS IN ADULT HUMAN ARTICULAR CARTILAGE
}

\author{
LEWIS B. LANE, PETER G. BULlOUGH
}

From the Hospital for Special Surgery, affiliated with the New York Hospital and Cornell University Medical College, New York

The thickness of the calcified zone of the articular cartilage and the number of tidemarks at the junction between articular cartilage and bone has been determined in specimens from 41 femoral heads and 42 humeral heads from cadavers aged between 25 and 93 . The thickness of the calcified zone decreased with age but the number of tidemarks increased, particularly over the age of 60 . These observations suggest that remodelling of the bone ends occurs and that this process is accelerated with increasing age.

Between the articular cartilage and the underlying bone is a narrow zone of calcified cartilage which appears to have two functions. It fastens the cartilage to the bone (Hough et al. 1974), and it provides for the growth and remodelling of the underlying bone by means of endochondral ossification, in which vascular invasion from the adjacent subchondral bone produces ossification of the calcified cartilage (Johnson 1959; Green et al. 1970).

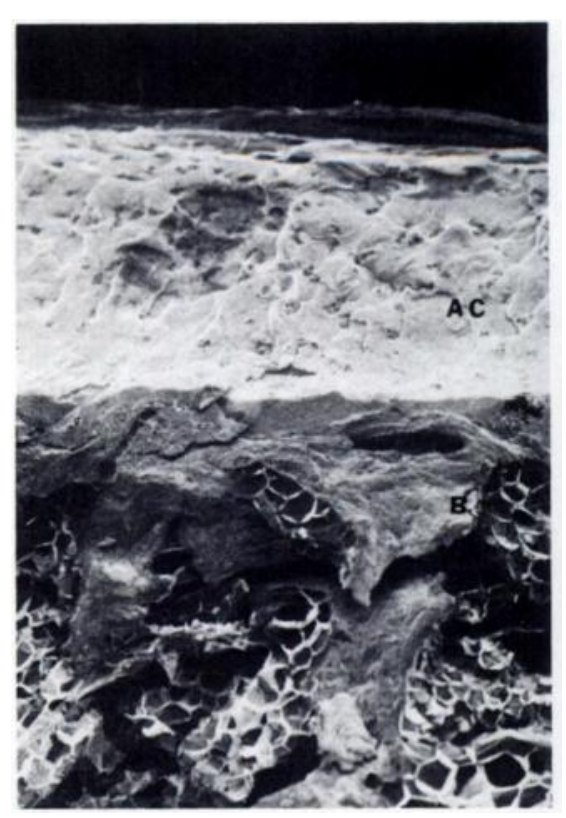

Fig. 1

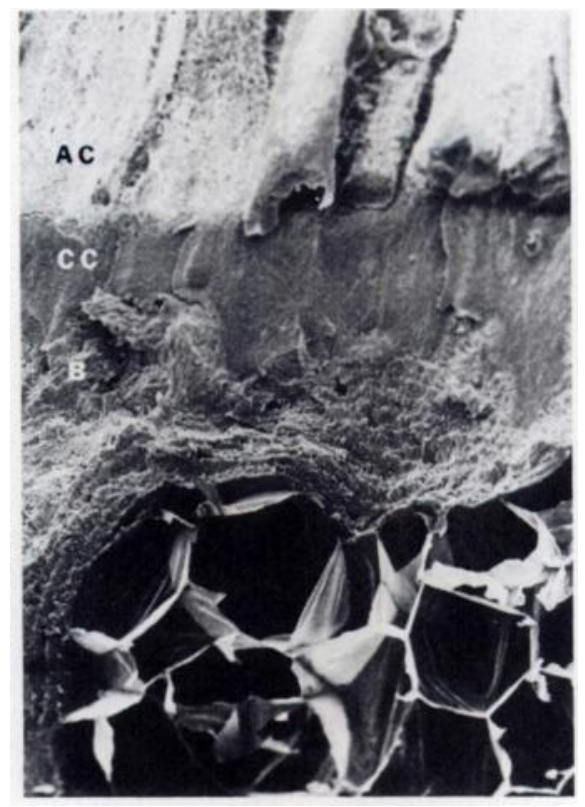

Fig. 2
Lane, Villacin and Bullough (1977) reported that the number of blood vessels and their modelling activity in the calcified zone varied with age and the patterns of loading within the joint. The number of vessels and the degree of modelling activity declined until the sixth or seventh decade after which they tended to rise to levels comparable to those found in younger subjects. More vessels and more active modelling were seen in the areas of the joint which were subjected to greater loading.

Figure 1 -Scanning electron micrograph of the end of a bone $(B)$ with overlying articular cartilage $(A C)$. $\times 7.5$. Figure $2-A$ close-up picture of Figure 1 to show the bone-cartilage interface. ( $B$, subchondral bone; $C C$, calcified cartilage; $A C$, articular cartilage.) $\times 50$. Figure 3-Photomicrograph of the junction of the calcified and non-calcified articular cartilage which shows here as a dark granular line, the tidemark $(T)$. Haematoxylin and eosin, $\times 150$.

L. B. Lane, MD, Orthopaedic Research Fellow \} The Hospital for Special Surgery,

P. G. Bullough, MD, Chief, Orthopaedic Pathology 335 East 70th Street, New York, NY 10021 , USA.

Requests for reprints should be sent to Dr P. G. Bullough. 


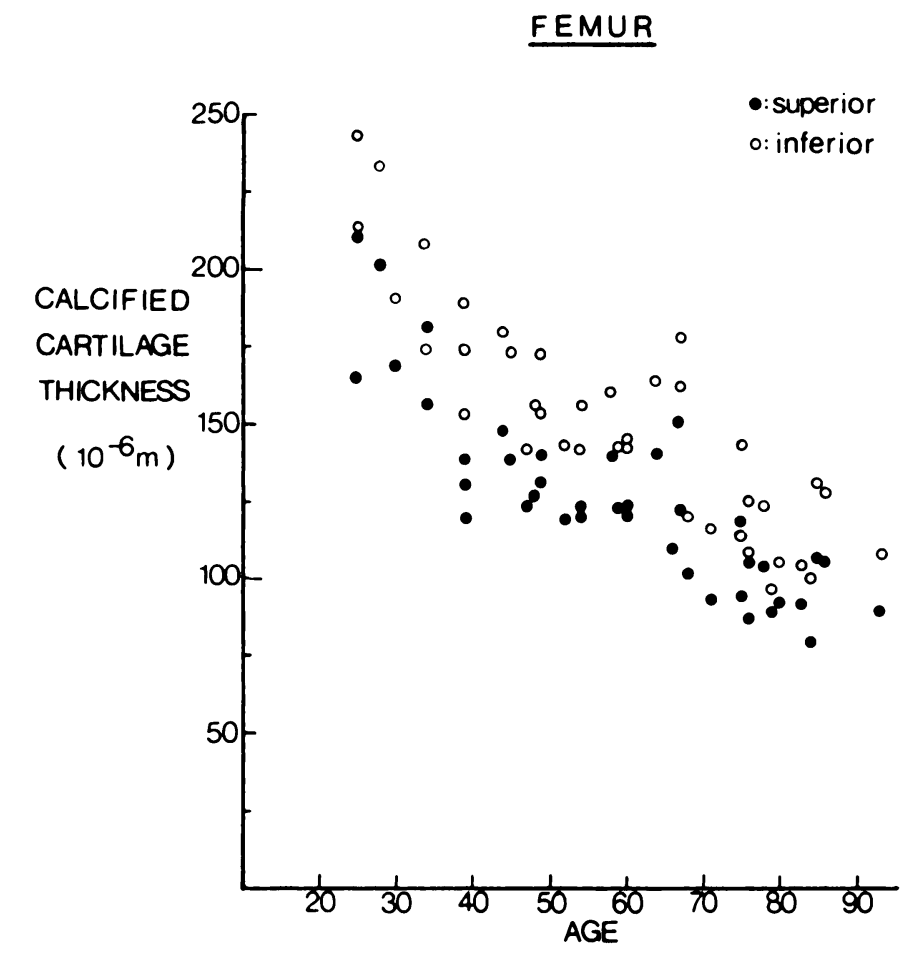

HUMERUS

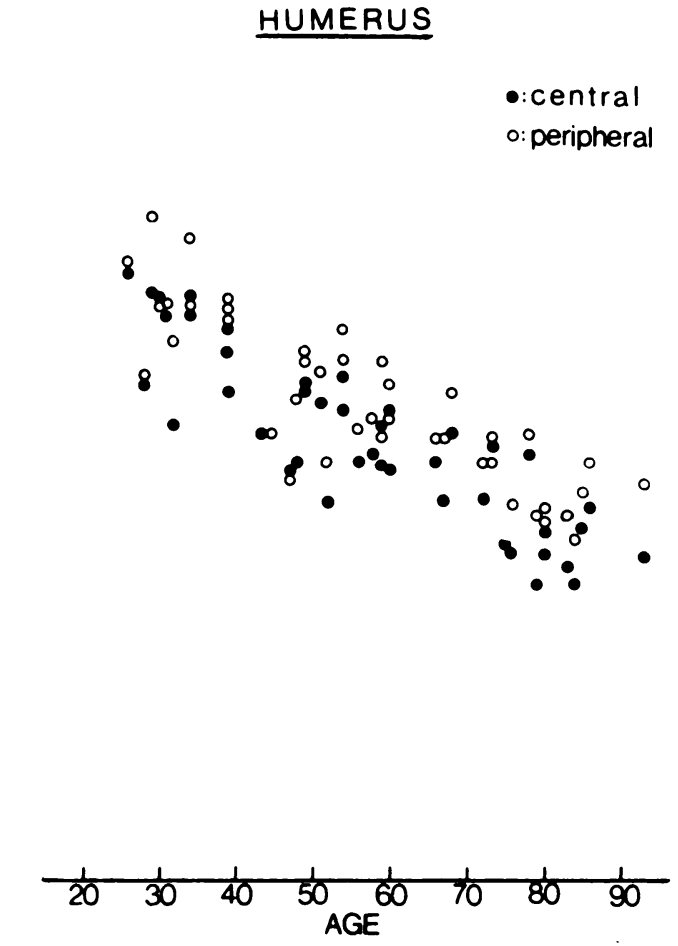

Fig. 4

The relation of the thickness of the layer of calcified cartilage to age.

Examination of sections stained with haematoxylin and eosin, through the junction between the articular cartilage and bone, show a basophilic line, the tidemark, separating the calcified from the uncalcified articular cartilage (Figs 1 to 3). Histochemical and tetracycline labelling studies of this zone have demonstrated that it is not an inert boundary but is metabolically active (Green et al. 1970; Lemperg 1971; Dmitrovsky, Lane and Bullough 1978). We have investigated the thickness of the calcified cartilage and the number of tidemarks present in sections of a number of specimens and evaluated the findings in respect of age and the distribution of joint loading.

\section{MATERIALS AND METHODS}

Forty-one femoral and $\mathbf{4 2}$ humeral heads were obtained from fresh amputation specimens or cadavers of human subjects aged from 25 to 93 years. The articular cartilage of each specimen and its respective opposing joint surface were examined grossly to confirm that the appearance was within normal limits for the age group (Heine 1926). Specimens showing obvious osteoarthritis were discarded from the study. Each specimen was assigned an arbitrary number and the code was broken only at the conclusion of the study in order to perform appropriate statistical analyses.

The distribution of the forces and patterns of loading through the hip and shoulder joints are well documented (Harrison, Schajowicz and Trueta 1953; Tobin 1955; Fessler 1957; Saha 1961; Frankel and Burstein 1970; Greenwald and O'Connor 1971; Greenwald and Haynes 1972; Poppen and Walker, personal communication). This information was used to define the portions of the heads of the humerus and femur which were subjected to the greatest and the least loading.

Each specimen was divided in sagittal section by a bandsaw into 6 to 10 blocks, each three millimetres thick. The blocks were then classified as being in the more or less heavily loaded portion of the femoral or humeral head, fixed in formalin, decalcified in 10 per cent nitric acid and embedded in paraffin. The blocks containing the intermediate boundary zone between the more or less heavily loaded areas were excluded in order to segregate clearly the portions of each head with different loading. Six to 10 sections, each five microns in thickness, were taken at different levels from the three to five blocks cut from each segment of each head. The sections were stained with haematoxylin and eosin and examined using a microscope with $a \times 16$ objective. Five approximately equidistant areas were chosen arbitrarily from each section for measurement and count of the tidemarks. The thickness of the calcified zone was determined by using a reference grid in the eyepiece to calculate the area, and dividing the derived area of the calcified cartilage by the length (DeHoff 1967). The number of tidemarks was counted at five equidistant points across each section and then averaged to obtain the number of tidemarks for that particular specimen.

\section{RESULTS}

\section{Thickness of the calcified zone}

Femoral head. The thickness of the calcified cartilage was from 79 to 243 microns, which agrees with the findings of other authors (Green et al. 1970; Stougård 1974). Figure 4 shows a steady decrease in the thickness of the calcified zone with increasing age of a magnitude of 60 per cent. The slope of the decrease in thickness is virtually the same for both the more stressed (superior) and less stressed (inferior) portions of the head. The calcified cartilage is approximately 10 to 15 per cent thicker in the less stressed areas in all ages $(P<0.02)$. Humeral head. The thickness of the calcified zone was from 96 to 206 microns. As shown in Figure 4 there is a 

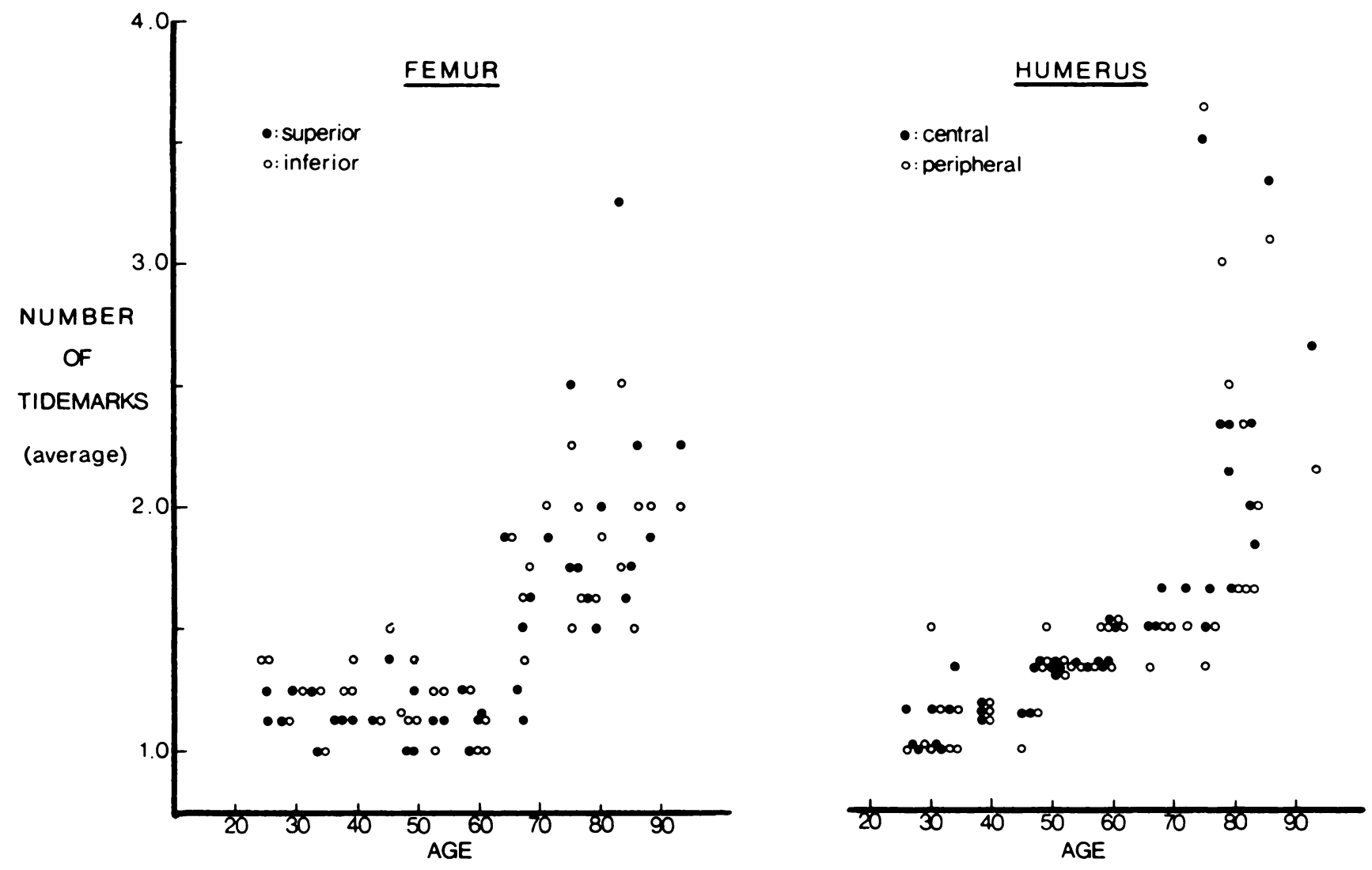

Fig. 5

The relation of the number of tidemarks to age.

steady decrease with advancing age, similar to that in the femoral head, though less steep and of narrower range. Again the slope of the decrease in thickness is virtually the same for both the more stressed (central) and less stressed (peripheral) portions of the head, while the calcified cartilage is found to be on average 5 to 10 per cent thicker in the less stressed portion of the head $(P<0.05)$. No statistically significant correlation was found between thickness and sex, race, or body weight.

\section{Number of tidemarks}

Femoral head. At least one tidemark was found to be present in all specimens observed and the average number in subjects aged 60 years and below ranged from 1.0 to 1.375 (with one exception which was 1.5 ). There was no obvious difference in number between areas of greater or lesser stress. After the age of 65 the average number increased, and after the age of 70 no specimens had less than an average of 1.5 tidemarks (Fig. 5).

Humeral head. The findings were similar to those in the femoral head. All specimens had at least one tidemark and no statistical difference in number was found between areas of greater or lesser stress. The average number was between 1.0 and 1.5 for specimens under the age of 60 , although a slightly upward trend was seen between the ages of 40 and 60 . The average number of tidemarks increased sharply after 60 . No statistically significant correlation was found between thickness and sex, race or body weight.

\section{DISCUSSION}

Calcified cartilage probably does not grow by interstitial growth. Changes in its thickness depend upon endochondral ossification, which results in addition to the subchondral bone and thinning of calcified cartilage, and on the incorporation of non-calcified articular cartilage into the calcified zone by advancement of the front of calcification, as shown by duplication of the tidemark, resulting in thickening of the calcified zone.

Previous studies have shown that endochondral ossification occurs continuously in the calcified zone (Johnson 1959; Green et al. 1970). The rate of this remodelling is variable, falling until the sixth decade and increasing thereafter (Lane et al. 1977). The number of tidemarks is just above unity until the age of 60 , when a sudden increase is observed. This should result in an increase in the thickness of the calcified zone of the cartilage but measurement shows that the calcified zone becomes thinner. The balance between the extension of the calcified zone into the non-calcified area of the articular cartilage and replacement by new bone via endochondral ossification at the bone-cartilage junc- 
tion must therefore be altered, with acceleration of endochondral ossification resulting in continual thinning of the calcified zone after the age of 60 . This change in bone dynamics may explain the changes which have been reported to occur in the geometry of the articular surface in later life (Goodfellow and Bullough 1967; Bullough, Goodfellow and O'Connor 1973; Freeman 1975).

\section{REFERENCES}

Bullough PG, Goodfellow JW, O'Connor J. The relationship between degenerative changes and load-bearing in the human hip.J Bone Joint Surg $[B r]$ 1973;55-B:746-58.

DeHoff RT. Sampling of material and statistical analysis in quantitative stereology. In: Elias H, ed. Proceedings of the 2nd International Congress for Stereology. Chicago, New York: Springer-Verlag, 1967:119-30.

Dmitrovsky E, Lane LB, Bullough PG. The characterization of the tidemark in human articular cartilage. Metabol Bone Dis Related Res 1978;1:115-8.

Fessler H. Load distribution in a model of a hip joint. J Bone Joint Surg [Br] 1957;39-B:145-53.

Frankel VH, Burstein AH. Orthopaedic biomechanics. Philadelphia: Lea \& Febiger, 1970.

Freeman MAR. The fatigue of cartilage in the pathogenesis of osteoarthritis. Acta Orthop Scand 1975;46:323-8.

Goodfellow JW, Bullough PG. The pattern of ageing of the articular cartilage of the elbow joint. J Bone Joint Surg [Br] 1967;49-B:175-81.

Green WT Jr, Martin GN, Eanes ED, Sokolof L. Microradiographic study of the calcified layer of articular cartilage. Arch Pathol 1970;90:151-8.

Greenwald AS, Haynes DW. Weight-bearing areas in the human hip joint. J Bone Joint Surg [Br] 1972;54-B:157-63.

Greenwald AS, O'Connor JJ. The transmission of load through the human hip joint. J Biomech 1971;4:507-28.

Harrison MHM, Schajowicz F, Trueta J. Osteoarthritis of the hip: a study of the nature and evolution of the disease. J Bone Joint Surg [Br] 1953;35-B:598-626.

Heine J. Uber die Arthritis deformans. Virchows Arch [Pathol Anat] 1926;260:521-663.

Hough AJ, Banfield WG, Mottram FC, Sokolof L. The osteochondral junction of mammalian joints. An ultrastructural and microanalytic study. Lab Invest 1974;31:685-95.

Johnson LC. Kinetics of osteoarthritis. Lab Invest 1959;8:1223-38.

Lane LB, Villacin A, Bullough PG. The vascularity and remodelling of subchondral bone and calcified cartilage in adult human femoral and humeral heads. J Bone Joint Surg [Br] 1977;59-B:272-8.

Lemperg $\mathbf{R}$. The subchondral bone plate of the femoral head in adult rabbits. I. Spontaneous remodelling studied by microradiography and tetracycline labelling. Virchows Arch [Pathol Anat] 1971;352:1-13.

Saha AK. Theory of shoulder mechanisms: descriptive and applied. Springfield, Illinois: Charles C. Thomas, 1961:11-14.

Stougård J. The calcified cartilage and the subchondral bone under normal and abnormal conditions. Acta Path Microbiol Scand Sect A 1974;82:182-8.

Tobin WJ. The internal architecture of the femur and its clinical significance. J Bone Joint Surg [Am] 1955;37-A:57-71. 